\title{
Molecular cloning and expression analysis of annexin A2 gene in sika deer antler tip
}

\author{
Yanling Xia ${ }^{1,2, *}$, Haomiao Qu ${ }^{1}$, Binshan Lu', Qiang Zhang ${ }^{1}$, and Heping Li ${ }^{1}$ *
}

\author{
* Corresponding Authors: \\ Yanling Xia \\ Tel: +86-0451-8219-0387, Fax: +86-0451-8219-0387, \\ E-mail: xiayanling@nefu.edu.cn \\ Heping Li \\ Tel: +86-0451-8219-0387, Fax: +86-0451-8219-0387, \\ E-mail: lihepinghrb2002@nefu.edu.cn \\ ${ }^{1}$ College of Wildlife Resources, Northeast Forestry \\ University, Harbin 150040, China \\ 2 Heilongjiang Academy of Agricultural Sciences \\ Postdoctoral Programme, Harbin 150086, China \\ ORCID \\ Yanling Xia \\ https://orcid.org/0000-0002-5407-9047 \\ Haomiao Qu \\ https://orcid.org/0000-0003-3758-7258 \\ Binshan Lu \\ https://orcid.org/0000-0002-4907-329X \\ Qiang Zhang \\ https://orcid.org/0000-0003-2996-8093 \\ Heping Li \\ https://orcid.org/0000-0001-8284-0449
}

Submitted Apr 11, 2017; Revised Jun 22, 2017; Accepted Aug 3, 2017
Objective: Molecular cloning and bioinformatics analysis of annexin A2 (ANXA2) gene in sika deer antler tip were conducted. The role of $A N X A 2$ gene in the growth and development of the antler were analyzed initially.

Methods: The reverse transcriptase polymerase chain reaction (RT-PCR) was used to clone the cDNA sequence of the ANXA2 gene from antler tip of sika deer (Cervus Nippon hortulorum) and the bioinformatics methods were applied to analyze the amino acid sequence of Anxa2 protein. The mRNA expression levels of the ANXA2 gene in different growth stages were examined by real time reverse transcriptase polymerase chain reaction (real time RT-PCR).

Results: The nucleotide sequence analysis revealed an open reading frame of 1,020 bp encoding 339 amino acids long protein of calculated molecular weight $38.6 \mathrm{kDa}$ and isoelectric point 6.09. Homologous sequence alignment and phylogenetic analysis indicated that the Anxa2 mature protein of sika deer had the closest genetic distance with Cervus elaphus and Bos mutus. Real time RT-PCR results showed that the gene had differential expression levels in different growth stages, and the expression level of the ANXA2 gene was the highest at metaphase (rapid growing period).

Conclusion: ANXA2 gene may promote the cell proliferation, and the finding suggested Anxa2 as an important candidate for regulating the growth and development of deer antler.

Keywords: Sika Deer; Annexin A2; Cloning; Phylogenetic Analysis; Real Time Reverse Transcriptase Polymerase Chain Reaction

\section{INTRODUCTION}

Deer antlers are bony appendages of the males in all deer species except for the reindeer (Rangifer tarandus). Deer antlers are the only mammalian appendages capable of repeated rounds of regeneration. Antler regeneration takes place in early spring each year, and antler rapid growth occurs during the late spring and summer. The annual regrowth and rapid growth of deer antlers are unique in adult mammals $[1,2]$. The developing antlers which are covered with velvet-like skin, are the fastest growing mammalian organs and the fastest growth rate can achieve 1 to $2 \mathrm{~cm}$ per day. Therefore, deer antlers offer a valuable model to study mechanisms of organ regeneration and rapid tissue growth [3,4].

Little is known of the factors involved in the development process of deer antlers. One of these factors may be annexin A2 (Anxa2), which is found in most eukaryotic cell types and is a calcium-dependent, phospholipid-binding protein, involved in regulating cellular functions. Anxa2 is proven to be associated with the occurrence, invasion and metastasis of cancers and has diverse roles in tumor development and progression. Over-expression of Anxa2 is observed in different types of tumors, such as pancreatic cancer, mammary cancer, while Anxa2 down-regulation has been associated with prostate cancer, esophageal squamous carcinoma and sinonasal adenocarcinoma [5-8]. 
Current literature shows that the proliferation rate of the deer antler tip tissue exceeds that of cancer cells. However, there are no reports regarding the effect of ANXA2 gene in the development process of deer antlers. In view of this, we cloned the sequence of the full coding region of ANXA2 gene, and studied its expression pattern in different antler growth stages. The putative protein sequence was characterized by comparing with other known species Anxa2 proteins and construction of a phylogenetic tree. Anxa2 expression levels during different growth stages were detected by using real time reverse transcriptase polymerase chain reaction (real time RT-PCR). These results provide basic data for further study of the ANXA2 gene's biological function and intrinsic regulation mechanism of deer antler growth at the molecular level.

\section{MATERIALS AND METHODS}

\section{Tissue samples}

Growing deer antler tips (about 30, 60, and 90 days) were used as source materials. Three male sika deer for each of the developmental stages were anaesthetized. The distal $5 \mathrm{~cm}$ of the antler tips was removed, and reserve mesenchyme was collected [9]. All the materials were prepared and immediately immersed into liquid nitrogen.

\section{Total RNA isolation}

Total RNAs were isolated using the Trizol reagent (Invitrogen, Shanghai, China) according to the manufacture's instruction. DNA was removed by incubating the total RNA with RNasefree DNase I (Promega, Shanghai, China) at $37^{\circ} \mathrm{C}$ for $30 \mathrm{~min}$. A spectrophotometer was used to detect the purity and concentration of the extracted RNAs. RNA integrity was determined by $1 \%$ agarose electrophoresis.

\section{Gene cloning and sequencing}

The extracted total RNAs were used to synthesize first-strand complementary DNA (cDNA) with an RT reagent kit (Toyobo, Beijing, China). One pair of primers was designed according to ANXA2 gene sequences of homologous species (F: 5'-TTCA AAATGTCTACCGTTCA-3'; R: 5'-AAACTAAACTAACAAA AGAGCG-3'). The PCR was performed using the above-mentioned primers and CDNA of rapid growing stage (60 days). PCR reaction mixture included $2.5 \mu \mathrm{L} 10 \times$ PCR buffer (with $\left.\mathrm{Mg}^{2+}\right), 2 \mu \mathrm{L}$ dNTP $(2.5 \mathrm{mM}), 1 \mu \mathrm{L}$ each of the primers ( $\left.10 \mathrm{mM}\right)$, $1 \mu \mathrm{L}$ template cDNA, $0.25 \mu \mathrm{L}$ Taq DNA polymerase (Takara, Dalian, China), finally adding sterile double distilled water to a total volume of $25 \mu \mathrm{L}$. PCR was conducted with the following thermocycles: $95^{\circ} \mathrm{C}$ for $5 \mathrm{~min} ; 40$ cycles of $95^{\circ} \mathrm{C}$ for $30 \mathrm{~s}$, $53^{\circ} \mathrm{C}$ for $1 \mathrm{~min}, 72^{\circ} \mathrm{C}$ for $1 \mathrm{~min}$; a final extension $72^{\circ} \mathrm{C}$ for 10 $\min$.

In the present study we obtained a PCR product of 1,180 nucleotides. The PCR product was separated on $1 \%$ agarose gel and purified with Gel Extraction Kit (Zoman, Beijing, China). Purified PCR products were then ligated into PMD18-T vector (Takara, China) and transformed into Escherichia coli. Positive clones were sequenced by two-tailed method.

\section{Bioinformatics analysis}

The obtained cDNA sequence was deduced to an amino acid sequence. Open reading frame of $A N X A 2$ gene was predicted with ORF search programs (http://www.ncbi.nlm.nih.gov/ gorf/gorf.html). The signal peptide site was predicted by SignalP3.0 (http://www.cbs.dtu.dk/services/SignalP-3.0/), and the Anxa2 protein molecular weight (MW) and isoelectric point (PI) were computed by applying the ProtParam tool (http:// web.expasy.org/protparam/). The Anxa2 protein sequence of sika deer was analyzed and compared using online BLASTP (http://www.ncbi.nlm.nih.gov/blast/). Conserved domain of Anxa2 protein was predicted by using InterProScan 5 (http:// www.ebi.ac.uk/Tools/pfa/iprscan5/). Two secondary structure and tertiary structure of the deduced amino acid sequence were predicted with GOR4 (https://npsa-prabi.ibcp.fr/cgi-bin/ secpred_gor4.pl) and SWISS-MODLE (http://swissmodel.expasy.org/). The multiple sequences alignment of Anxa2 proteins was performed with DNAStar7.10. A phylogenetic tree was constructed using MEGA 6.0.

\section{Expression analysis of $A N X A 2$ gene}

Quantitative real time reverse transcriptase PCR (qRT-PCR) was used to detect the mRNA expression levels of ANXA2 gene in different growth stages. Reactions of qRT-PCR were carried out using Chromo 4 Real-Time PCR Detector (MJ Research, Shanghai, China) with SYBR Premix EX TaqTM II (Takara, China). In the real time RT-PCR study, specific primers (AnxA 2-F: 5'-CGTTTCCGACACATCTGGC-3' and AnxA2-R: 5'CCCTGGCATCCTGGTCAAT- ${ }^{\prime}$ ) were used to amplify a 113 bp fragment with cDNA from reserve mesenchyme of the different growth stages. The housekeeping gene $\beta$-actin was used as an internal reference for data normalization ( $\beta$-actin-F: 5'-GCGTGACATCAAGGAGAAGC- ${ }^{\prime}$ and $\beta$-actin-R: $5^{\prime}$ GGAAGGACGGCTGGAAGA-3', 173 bp). The PCR system $(25 \mu \mathrm{L})$ consisted of $12.5 \mu \mathrm{L} 2 \times$ SYBR Green Master Mix, 1.5 $\mu \mathrm{L}$ (each) forward primer and reverse primer $(10 \mathrm{mM}), 2 \mu \mathrm{L}$ template, and $7.5 \mu \mathrm{L}$ ddH2O. The thermal profile for SYBR Green real time PCR was $95^{\circ} \mathrm{C}$ for $30 \mathrm{~s}$, followed by 40 cycles of $95^{\circ} \mathrm{C}$ for $30 \mathrm{~s}$, and $60^{\circ} \mathrm{C}$ for $30 \mathrm{~s}$, and $72^{\circ} \mathrm{C}$ for $1 \mathrm{~min}$. All sample was run in triplicate. The expression levels of the target genes were normalized with the reference $\beta$-actin and calculated using the $2^{-\Delta \Delta \mathrm{CT}}$ method [10]. The sample of $30 \mathrm{~d}$ was used as the control group and the samples of $60 \mathrm{~d}$ and $90 \mathrm{~d}$ were used as the experimental groups.

\section{RESULTS AND DISCUSSION}




\section{Sequencing and bioinformatics analysis of Anxa2}

The full-length sequence of the obtained sika deer ANXA2 gene was $1,180 \mathrm{bp}$, including a complete $1,020 \mathrm{bp}$ open reading frame flanked by 6 bp 5' untranslated region (UTR) and 154 bp 3' UTR. ANXA2 gene of sika deer encodes a polypeptide of 339 amino acids with a deduced MW of $38.6 \mathrm{kDa}$ and an $\mathrm{PI}$ of 6.09 (Figure 1). The Anxa2 peptide was predicted to have no $\mathrm{N}$-terminal signal peptide. The secondary structure prediction of Anxa2 protein indicated that $46.61 \%$ alpha helix, $14.75 \%$ extended chain, and $38.64 \%$ random coil might be formed (Figure 2). Like other Annexins, Anxa2 protein of sika deer had the typical structure of Annexin, containing a highly conserved C-terminal core domain and a relative flexible $\mathrm{N}$-terminal domain [11]. C-end domain contained four highly conserved Annexin domains at amino acids 37 to 102, 109 to 174,193 to 259 , and 269 to 334 (Figure 3), which is a $\mathrm{Ca}^{2+}$, phosphorus lipid and actin binding site. $\mathrm{N}$ terminal was its functional region, which contained Tyr23, Ser11, Ser25 phosphorylation site and a nuclear export signal [12]. Phosphorylation is very important for the interaction of Anxa2 and

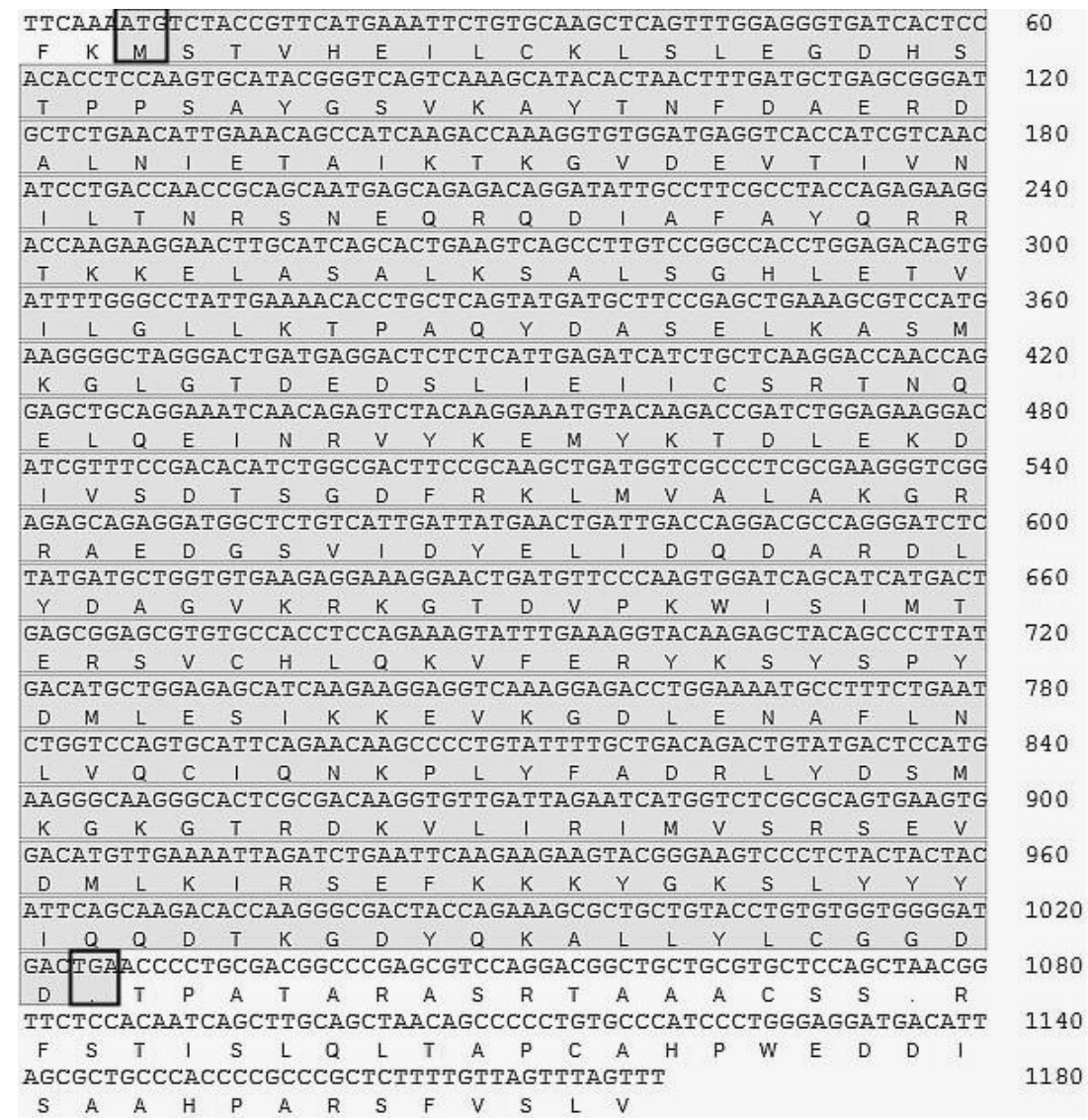

Figure 1. Nucleotide and deduced amino acid sequence of annexin A2 cDNA from sika deer (ATG is the initiation codon; * is the stop codon).

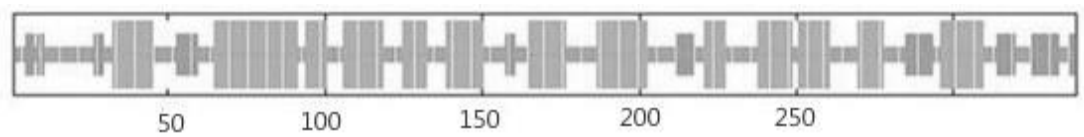

Figure 2. Predicted secondary structure of the sika deer annexin A2 protein by GOR4. Alpha helix, extended strand, random coil are indicated, respectively, with the longest, the second longest, the third longest.

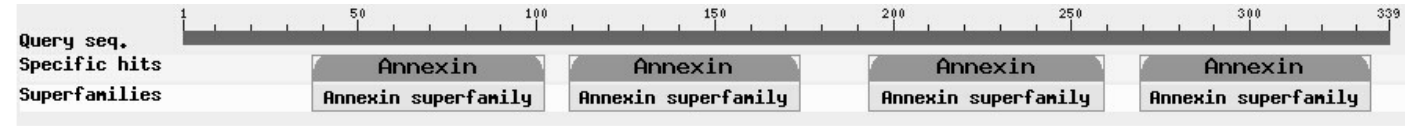

Figure 3. Conserved domain prediction of annexin A2 protein from sika deer. 
related proteins, and it is crucial for nucleo-cytoplasmic transport.

Amino acids sequence of sika deer Anxa2 protein was compared with Bos mutus (NP_777141.1), Canis lupus familiaris (NP_001002961.1), Cervus elaphus (Q2Q1M6.1), Sus scrofa (NP_001005726.1), Mus musculus (NP_031611.1), Homo sapiens (AAH23990.1), Gallus gallus (NP_990682.1), and the consistency of amino acids was from 95\% to 98\% (Figure 4). Homological analysis showed that Anxa2 proteins derived from different species shared high homology in evolution. High conservation of Anxa2 proteins shows that the function of ANXA2 gene in different species is very similar. It implies that ANXA2 gene is an important stabilizing agent for cell functions.

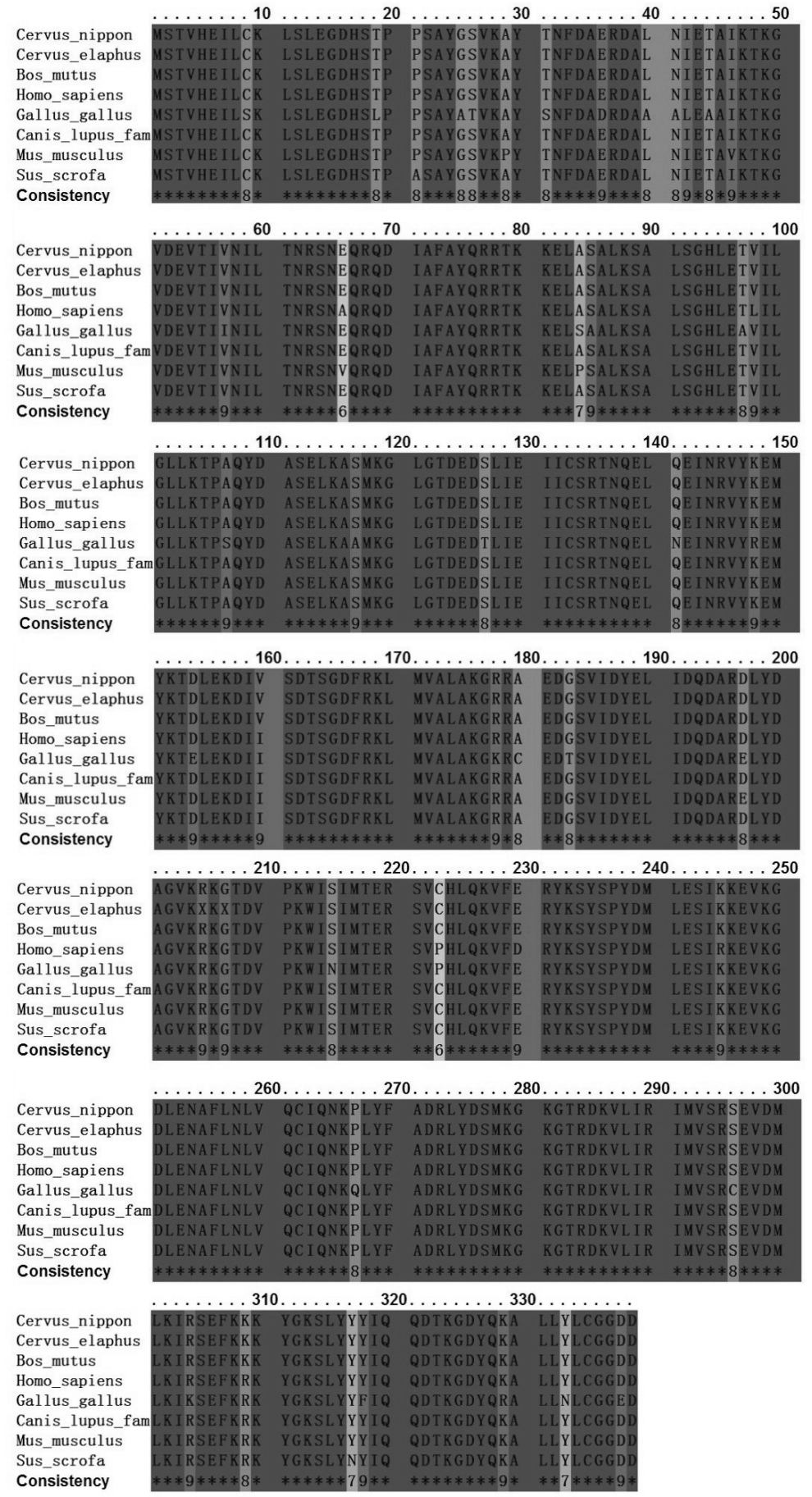

Figure 4. Amino acid sequences comparison of annexin A2 proteins from different species. The peptide of annexin A2 was compared with the corresponding sequences from Bos mutus, Canis lupus familiaris, Cervus elaphus, Sus scrofa, Mus musculus, Homo sapiens, and Gallus gallus. Conserved sequences are indicated by ( ${ }^{*}$ ), and the variant residues were listed. 
Based on the amino acid sequences of Anxa2 proteins, a phylogenetic tree was constructed using the neighbor-joining method (Figure 5). The result showed that Anxa2 protein of sika deer was most related to Cervus elaphus and Bos mutus, whereas it was most distant from Gallus gallus. The analysis result was consistent with the status of the various species in the traditional taxonomy.

\section{Expression pattern of $A N X A 2$ gene in different growth stages}

The mRNA expression levels of the ANXA2 gene were examined by real time PCR in different growth stages, including early growth stage (about $30 \mathrm{~d}, \mathrm{~S} 1$ ), mid growth stage (about 60 d, S2), late growth stage (about 90 d, S3). As shown in Figure 6. ANXA2 gene was expressed in all three stages, while the AnxA2 mRNA expression level was highest at S2 stage. Deer antler growth process is divided into a growth period and an ossification period. In the rapid growing period (about $60 \mathrm{~d}$ ) the maximal elongation rate of deer antler can be up to 1 to 2 $\mathrm{cm} / \mathrm{d}$, more than the growth rate of any mammal bone tissue. In the present study the gene has a higher expression level in S2 stage (1.98) than in other stages S1 (1.0), S3 (0.66), and the relative expression of $\mathrm{S} 2$ was about 2 and 3 times that of $\mathrm{S} 1$ and S3.

Current literature suggests that ANXA2 gene is involved in the regulation of epidermal growth factor receptor (EGFR) signaling pathway. EGFR is widely distributed in normal and malignant epithelial cells, and its overexpression and self-activation can promote cell proliferation and differentiation. Anxa2 is recognized to act as the downstreamer of EGFR signaling in breast cancer cells $[13,14]$. The blocked Anxa2 or the Anxa2 knockdown inhibits the activation of the EGFR downstream pathways and reduces breast cancer cell migration [15].

Anxa2 mRNA expression level is the highest in reserve mesenchyme region of different tissue layers (reserve mesenchyme, precartilage, cartilage) of deer antler tip [16]. Thus in the present study we selected the reserve mesenchyme tissue as research materials. The result showed that $A N X A 2$ gene had higher expression level at S2 (fast growing period). Anxa2 mRNA up-regulated from S1 to S2 and down-regulated form S2 to S3. We speculate that higher expression of ANXA2 gene

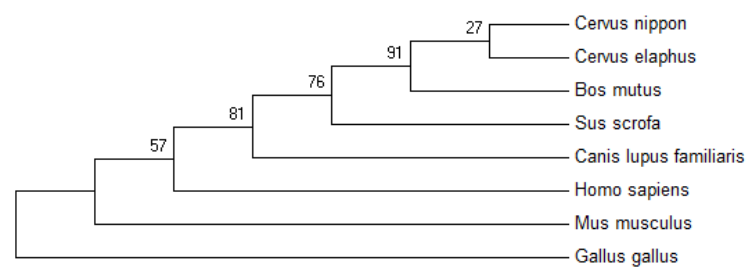

Figure 5. The phylogenetic tree of annexin A2 proteins from different species. Phylogenetic analysis based on the annexin $A 2$ amino acid sequences from various animals. The phylogenic tree of the annexin A2 proteins was constructed using the neighbor-joining method.

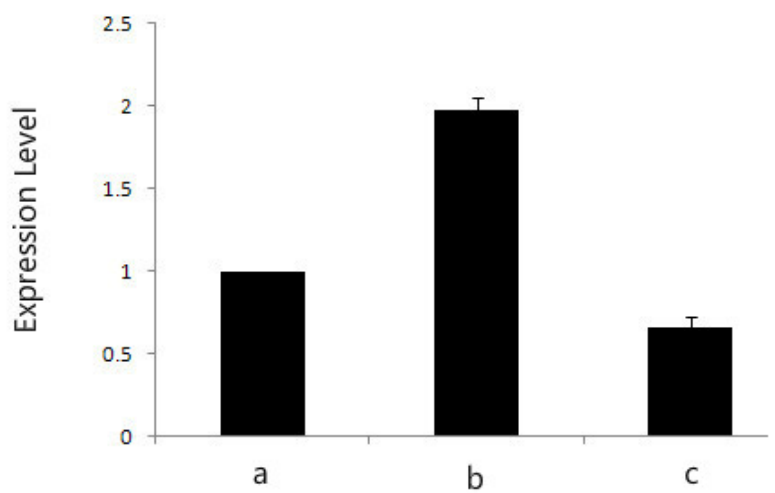

Figure 6. Expression levels of annexin A2 gene in different growth stages. (a) Deer antler of $30 \mathrm{~d}$. (b) Deer antler of $60 \mathrm{~d}$. (c) Deer antler of $90 \mathrm{~d}$.

in rapid growing period can be involved in the EGFR signaling pathway, which promote cell proliferation. However expression down-regulation of ossification period (about 90d) may be related to the slow growth and fast ossification of S3. Real time RT-PCR testing results indicated that Anxa2 may play an important role in regulating rapid growth of deer antler in the growth period. To further study the function of ANXA2 gene, our laboratory is constructing an eukaryotic expression vector of ANXA2 gene to transfect mesenchymal cells of sika deer antlers.

\section{CONFLICT OF INTEREST}

We certify that there is no conflict of interest with any financial organization regarding the material discussed in the manuscript.

\section{ACKNOWLEDGMENTS}

This research was supported by Fundamental Research Funds for the Central Universities (No.2572014CA01) and China Postdoctoral Science Foundation (No. 20110491124).

\section{REFERENCES}

1. Price JS, Allen S, Faucheux C, Althnaian T, Mount JG. Deer antlers: a zoological curiosity or the key to understanding organ regeneration in mammals? J Anat 2005;207:603-18.

2. Li CY, Yang FH, Li GY, et al. Antler regeneration: a dependent process of stem tissue primed via interaction with its enveloping skin. J Exp Zool 2007;307:95-105.

3. Brockes JP, Kumar A. Appendage regeneration in adult vertebrates and implications for regenerative medicine. Science 2005;310:1919-23.

4. Lord EA, Martin SK, Gray JP, Li CY, Clark DE. Cell cycle genes PEDF and CDKN1C in growing deer antlers. Anat Rec (Hoboken) 2007;290:994-1004. 
5. Zhang, F, Wang ZY, Yuan J, et al. RNAi-mediated silencing of Anxa2 inhibits breast cancer cell proliferation by downregulating cyclin D1 in STAT3-dependent pathway. Breast Cancer Res Treat 2015;153:263-75.

6. Maule F, Bresolin S, Rampazzo E, et al. Annexin 2A sustains glioblastoma cell dissemination and proliferation. Oncotarget 2016;7:54632-49.

7. Lokman NA, Ween MP, Oehler MK, Ricciardelli C. The role of annexin A2 in tumorigenesis and cancer progression. Cancer Microenviron 2011;4:199-208.

8. Zhang X, Liu S, Guo C, Zong J, Sun MZ. The association of annexin A2 and cancers. Clin Transl Oncol 2012;14:634-40.

9. Li CY, Clark DE, Lord EA, Stanton JA, Suttie JM. Sampling technique to discriminate the different tissue layers of growing antler tips for gene discovery. Anat Rec 2002;268:125-30.

10. Livak KJ, Schmittgen TD. Analysis of relative gene expression data using real-time quantitative PCR and the $2^{-\Delta \Delta C \mathrm{~T}}$ method. Methods 2001;25:402-8.

11. Jost M, Gerke V. Mapping of a regulatory important site for protein kinase $\mathrm{C}$ phosphorylation in the $\mathrm{N}$-terminal domain of annexin II. Biochim Biophys Acta 1996;1313:283-9.

12. Liu J, Rothermund CA, Ayala-Sanmartin J, Vishwanatha JK. Nuclear annexin II negatively regulates growth of LNCaP cells and substitution of ser 11 and 25 to glu prevents nucleo-cytoplasmic shuttling of annexin II. BMC Biochem 2003;4:10.

13. Grewal T, Enrich C. Annexins-modulators of EGF receptor signalling and trafficking. Cell Signal 2009;21:847-58.

14. Ackland ML, Newgreen DF, Fridman M, et al. Epidermal growth factor-induced epithelio-mesenchymal transition in human breast carcinoma cells. Lab Invest 2003;83:435-48.

15. Shetty PK, Thamake SI, Biswas S, Johansson SL, Vishwanatha JK. Reciprocal regulation of annexin A2 and EGFR with Her-2 in Her-2 negative and herceptin-resistant breast cancer. PLoS ONE 2012;7(9):e44299.

16. Molnár A, Gyurján I, Korpos E, et al. Identification of differentially expressed genes in the development antler of red deer (Cervus elaphus). Mol Genet Genomics 2007;277:237-48. 